\title{
Uso do perioguide na cirurgia para correção do sorriso gengival
}

\author{
The use off perioguide in surgery to correct gingival smile \\ Uso de perioguide en cirugía para la corrección de sonrisa gingival
}

\section{Resumo}

O padrão estético exige sorrisos bonitos e harmoniosos, o que incentiva a procura por procedimentos odontológicos para correção de imperfeições no sistema estomatognático. Na periodontia as cirurgias plásticas com intuito de corrigir o chamado sorriso gengival vêm sendo cada vez mais rotineiras. Com o advento de novas tecnologias, aumentam as opções de tratamento estético que se tornaram cada vez menos invasivos, menos traumáticos e com maior previsibilidade, possibilitando ênfase neste âmbito. Nesse sentido, toma-se o perioguide como objeto de pesquisa, uma vez que o que o mesmo se propõe a auxiliar o cirurgião-dentista na previsibilidade na cirurgia de correção do sorriso gengival. Este trabalho trata-se de um estudo de caso único, pois possui a intenção de relatar um trabalho que trata cirurgia de gengivoplastia/gengivectomia com utilização do perioguide. Concluiu-se que a atuação interdisciplinar entre as especialidades da Periodontia com a Radiologia auxiliada com a tecnologia possibilita aperfeiçoar e otimizar a cirurgia de gengivoplastia/gengivectomia. O uso do perioguide foi um diferencial para sucesso do tratamento.

Palavras-chave: Periodontia; Cirurgia Bucal; Sorriso.

\begin{abstract}
The aesthetic standard requires beautiful and harmonious smiles, which encourages the search for dental procedures to correct imperfections in the stomatognathic system. In periodontics, plastic surgeries with the aim of correcting the socalled gummy smile are becoming progressively routine. With the advent of new technologies, aesthetic treatment options increase, becoming less invasive, less traumatic and with greater predictability, enabling emphasis in this area. In this sense, the perioguide is taken as an object of research, since its purpose is to help dentists in predicting gummy smile correction surgery. This work is a single case study, as it intends to report a work dealing with gingivoplasty/gingivectomy surgery using the perioguide. The interdisciplinary work between the specialties of Periodontics was concluded, with Radiology aided by technology, which enables the improvement and optimization of gingivoplasty/gingivectomy surgery. The use of the perioguide was a differential for treatment success.
\end{abstract}

Keywords: Periodontics; Surgery, Oral; Smiling. 


\section{Resumen}

El estándar estético requiere sonrisas hermosas y armoniosas, lo que incentiva la búsqueda de procedimientos dentales para corregir imperfecciones en el sistema estomatognático. En periodoncia, las cirugías plásticas con el objetivo de corregir la llamada sonrisa gingival son cada vez más rutinarias. Con el advenimiento de las nuevas tecnologías, aumentan las opciones de tratamiento estético que se han vuelto cada vez menos invasivas, menos traumáticas y con mayor previsibilidad, lo que permite hacer hincapié en esta área. En este sentido, la perioguía se toma como objeto de investigación, ya que su finalidad es ayudar a los odontólogos en la predicción de la cirugía de corrección de la sonrisa gingival. Este trabajo es un estudio de caso único, ya que pretende informar de un trabajo sobre la cirugía de gingivoplastia / gingivectomía utilizando la perioguía. Se concluyó el trabajo interdisciplinario entre las especialidades de Periodoncia, con Radiología asistida por tecnología, que permite la mejora y optimización de la cirugía de gingivoplastia / gingivectomía. El uso de la perioguía fue un diferencial para el éxito del tratamento.

Palabras clave: Periodoncia; Cirugía Bucal; Sonrisa.

\section{Introdução}

Na periodontia, o fluxo digital vem se tornando cada vez mais cotidiano e com o advento de novas tecnologias, aumentam as opções de cirurgias de correção do sorriso gengival. De acordo com Kahn e Dias (2017, p. 4),

O sorriso gengival é uma entidade clínica extremamente complexa, que apresenta diversas etiologias e, sendo assim, alguns tipos de tratamentos. Pode ser definido como uma alteração estética relativamente frequente caracterizada pela exposição excessiva das gengivas durante o movimento do lábio superior para o sorriso. É o resultado de uma relação inadequada entre a borda inferior do lábio superior, o posicionamento dos dentes anteriores superiores, a localização da maxila e a posição da margem gengival em relação à coroa dentária.

Segundo Joly (2010), a composição de um sorriso considerado belo, atraente e saudável, envolve o equilíbrio entre a forma e a simetria dos dentes, lábios e gengiva, além da maneira como eles se relacionam com a face dos pacientes. Pesquisas recentes indicam que utilizando o guia cirúrgico periodontal é possível um tratamento cada vez menos invasivo, menos traumático e com maior previsibilidade, melhorando a comunicação com o paciente. Nesse sentido, toma-se o perioguide como objeto de pesquisa, uma vez que o mesmo tem objetivo de auxiliar o cirurgião-dentista na previsibilidade da cirurgia de correção do sorriso gengival.

A cirurgia de correção é tradicionalmente realizada a partir da sondagem periodontal e demarcação manual do tecido, seguida da incisão primária para a remoção do colarinho gengival com cabo de bisturi e lâmina, aumentando o risco de falhas como a remoção excessiva de tecido gengival e ósseo, resultando em recessão gengival seguida de sensibilidade dental, devido a exposição da raiz. Visto isso, com o auxílio da tecnologia, o guia cirúrgico periodontal (perioguide) busca tornar o procedimento mais seguro para o cirurgião-dentista e para o paciente. (Nunes et al. 2020).

A etimologia da palavra "perioguide", por influência do inglês guide, que significa guia com perio que remete à especialidade odontológica que tem como objetivo o estudo dos tecidos de suporte e circundantes dos dentes e seus substitutos. O mesmo foi projetado utilizando o software de planejamento Exocad, tendo como base a união do arquivo em .STL através do escaneamento intraoral (3Shape) com o arquivo.DICOM da tomografia computadorizada de feixe cônico com afastamento de lábios. De acordo com a metodologia proposta por Januário (2008), é possível mensurar toda dimensão gengival, além dos tecidos duros, utilizando um simples afastador labial, deste modo avalia-se com precisão o fenótipo gengival do paciente, que são classificados segundo o estudo de Zweers et al. (2014) em três tipos:

1. fenótipo festonado em que há maior associação com coroa triangular delgada, convexidade cervical sutil, contatos interproximais próximos à borda incisal e uma zona estreita de tecido queratinizado, gengiva delicada e fina e um osso alveolar relativamente fino; 
2. fenótipo liso espesso mostrando coroas dentárias mais em forma de quadrado, pronunciada convexidade cervical, grande contato interproximal localizado mais apicalmente, uma zona ampla de tecido queratinizado, gengiva fibrosa espessa e um osso alveolar relativamente grosso;

3. fenótipo espesso festonado possui gengiva fibrótica espessa, dentes finos, zona estreita de tecido queratinizado e pronunciado recorte gengival.

A partir do planejamento, o guia é extraído do mundo virtual através de uma impressão tridimensional (3D) utilizando uma resina biocompatível que possibilita seu uso nas cirurgias periodontais de maneira segura e personalizada. Com o planejamento virtual garante-se uma reabilitação estética e funcional, recuperação pós-operatória menos dolorosa e melhor comunicação com o paciente, atendendo assim, suas expectativas.

\section{Metodologia}

Este trabalho trata-se de um estudo de caso único, pois possui a intenção de relatar uma cirurgia de correção do sorriso gengival, isto é, gengivoplastia/gengivectomia com utilização do perioguide. Sendo tratado em um estudo qualitativo, verificando e possibilitando a resolução das problemáticas solicitadas pela paciente. Este estudo foi realizado através uma anamnese criteriosa onde o paciente de 31 anos (M.I.C.B.R.), procurou a clínica da disciplina de Periodontia II do Centro Universitário Fluminense - UNIFLU/FOC, relatando insatisfação estética devido ao sorriso gengival, onde há uma exposição excessiva de gengiva inserida durante o movimento dos lábios superiores para o sorriso. A paciente examinada assinou o Termo de Consentimento Livre e Esclarecido (TCLE), autorizando a realização de registros de fotografias e análises dos prontuários. Associando este estudo de caso, fez - se uma pesquisa literária em banco de dados como Pubmed, BVS e CAPES, realizando uma análise nos artigos que estavam vinculados à temática do estudo, auxiliando ao decorrer do procedimento.

\section{Relato de caso}

No exame clínico nos mostrou dentes anteriores superiores curtos e com excesso de tecido gengival, o que determinou o planejamento de um aumento de coroa clínica nos dentes anteriores superiores do 15 ao 25, através de uma genioplastia com bisel interno e osteotomia para devolução do espaço supracrestal. $\mathrm{O}$ exame periodontal apresentou profundidades de sondagens 2-3 mm com níveis adequados de saúde periodontal, isto é, sem presença de placa, sangramento ou bolsa periodontal. Foram realizadas fotografias da paciente com sorriso forçado (Figura 1) e do aspecto intrabucal (Figura 2).

Figura 1 - Sorriso evidenciando sorriso gengival.

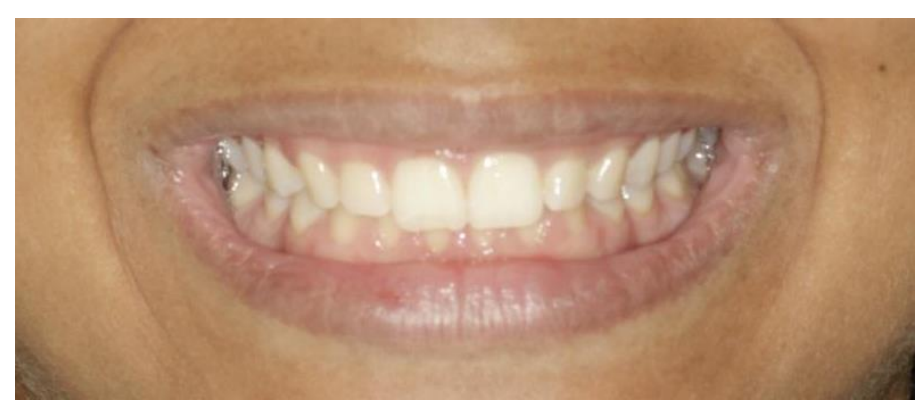

Fonte: Autores. 
Figura 2 - Inicial Intrabucal.

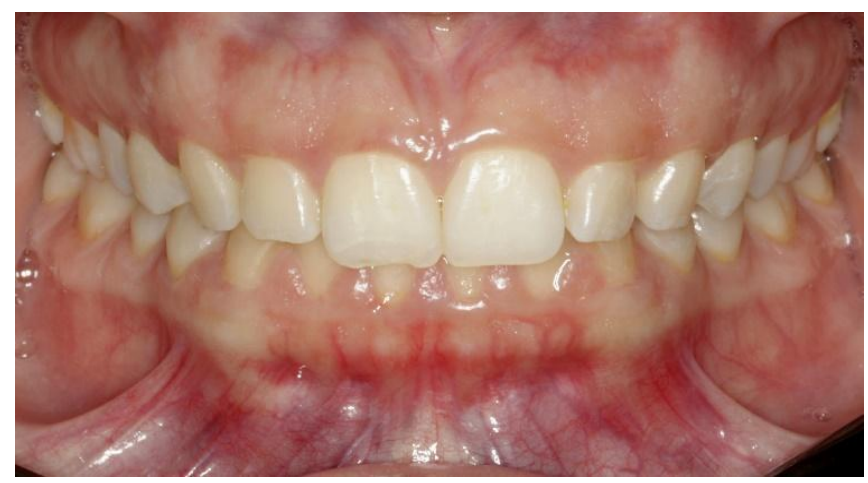

Fonte: Autores.

Após o exame clínico e periodontal pode-se observar erupção passiva alterada, com isso foi necessário o uso da tomografia computadorizada de feixe cônico com afastamento de tecidos moles seguindo a metodologia de Januário et al. (2008) e escaneamento intraoral por software digital para obtenção de um modelo digital em .STL (3Shape, Copenhagen K, Dinamarca).

Inicialmente no plano de tratamento foi estabelecido um protocolo de medidas tomográficas onde foi determinada a quantidade de tecido ósseo e gengival a ser removido. Nesse protocolo foi-se mensurado distâncias: da junção cementoesmalte (JCE) à margem gengival, que vai determinar a quantidade de tecido mole a ser removida; da JCE à crista óssea, que determinará a necessidade de recontorno ósseo; a distância da margem gengival à crista óssea; e a espessura gengival, para avaliar o fenótipo gengival e o tamanho das coroas anatômicas.

A altura do zênite do sorriso foi demarcada: o canino deve ser 0,5 a $1 \mathrm{~mm}$ maior que os incisivos centrais e os incisivos laterais 0,5 a $1 \mathrm{~mm}$ abaixo do que os incisivos centrais. A posição da papila interdental deve estar além do ponto de contato interproximal, fechando o espaço interdental, para promover um sorriso mais harmônico. Finalmente, a proporção estética, altura x largura, dos últimos elementos são verificados. O tamanho dos incisivos centrais baseia-se na proporção ideal para proporcionar uma estética agradável de sorriso, entre $9,5 \mathrm{~mm}$ e $11 \mathrm{~mm}$.

Em seguida, as medidas obtidas na tomografia foram transferidas para o Exocad, onde é possível fazer uma sobreposição com as imagens em STL adquiridas com o escaneamento. O caso foi encaminhado para o Planning Center, onde realizou-se o planejamento digital e a confecção do guia duplo periodontal (perioguide) com a borda superior do guia com 2,5 mm em toda sua extensão.

A prototipagem do perioguide foi realizada em impressora 3D de estereolitografia Formlabs 2TM (Somerville, Massachusetts, EUA) com resina SG $^{\mathrm{TM}}$ biocompatível, seguindo os passos de pós-processamento com imersão em álcool isopropílico e sob exposição de luz UV ambos por 20 minutos. O objetivo é secar a peça e aumentar a resistência mecânica da mesma. A limpeza final foi feita com o auxílio de uma cuba ultrassônica e detergente neutro. Após a secagem é feita a embalagem e finaliza-se o processo de fabricação da guia de perioguide (Figura 3). 
Figura 3 - Guia Perioguide.

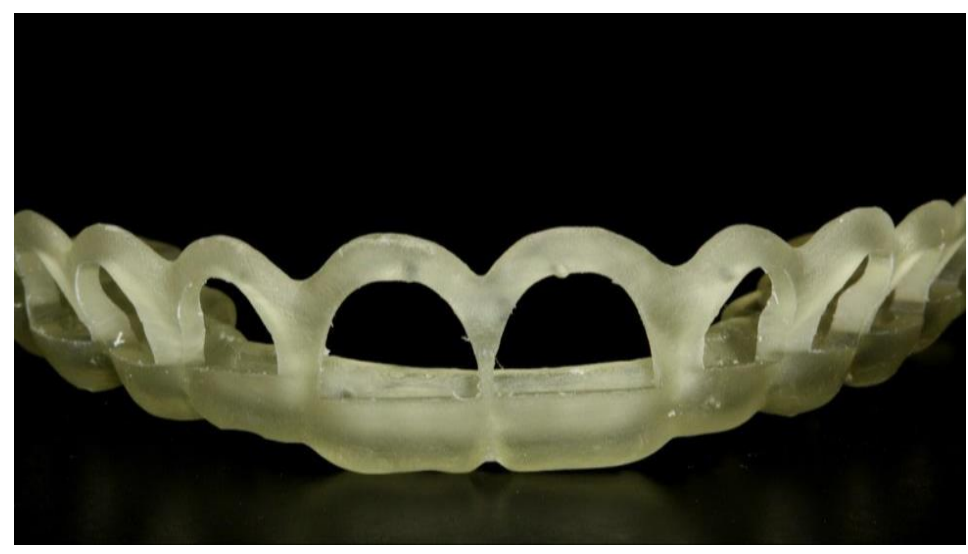

Fonte: Autores.

Na fase pré-operatória, foi solicitado à paciente que fizesse uso de amoxicilina 500 mg, 04 cápsulas e decadron 04 mg 01 cápsula, ambas 01 hora antes do procedimento. Na fase cirúrgica, inicialmente, a assepsia extra oral foi realizada com álcool $70 \%$ e a paciente foi solicitada a realizar bochecho com solução de clorexidina a 0,12\% por 1 minuto (Souza et al. 2020). Em sequência, com técnica anestésica infiltrativa local com lidocaína 2\% com epinefrina 1:100.000 ( Nova DFL, Industry e Commerce SA, Rio de Janeiro, Brasil) foi administrado na parte inferior do vestíbulo maxilar.

Após posicionamento do Perioguide (Figura 4), usando um milímetro de sonda (Hu-Friedy, Chicago, EUA), a JCE foi localizada e em comparação com a delimitação do guia cirúrgico posicionado em boca. As incisões para remoção do excesso gengival foram realizadas com uma lâmina de bisturi 15C em aço carbono (Swann-Morton, Inglaterra) em bisel interno de $45^{\circ}$ em relação à gengiva iniciando no elemento 21 indo até o elemento 25 e em seguida do elemento 11 ao 15 (Figura 5), removendo o excesso gengival (Figura 6) a cada incisão com uma cureta gracey 5-6 e refinando o corte com tesoura castroviejo. Verificando a profundidade óssea de sondagem após a remoção do excesso gengival, estes apresentavam apenas $2 \mathrm{~mm}$, tendo então que abrir um retalho de Neumam para assim realizar a osteotomia com broca 2173ESP (KG Sorensen, Brasil) acompanhando a anatomia dos dentes para a devolução do espaço biológico periodontal (Figura 7), que com a nova aferição apresentou 3 mm de profundidade de sondagem. Para as suturas do tipo suspensório foram utilizados fios agulhados 4-0 com auxílio do porta agulha do tipo castroviejo (Figura 8 e 9).

Figura 4 - Perioguide Posicionado.

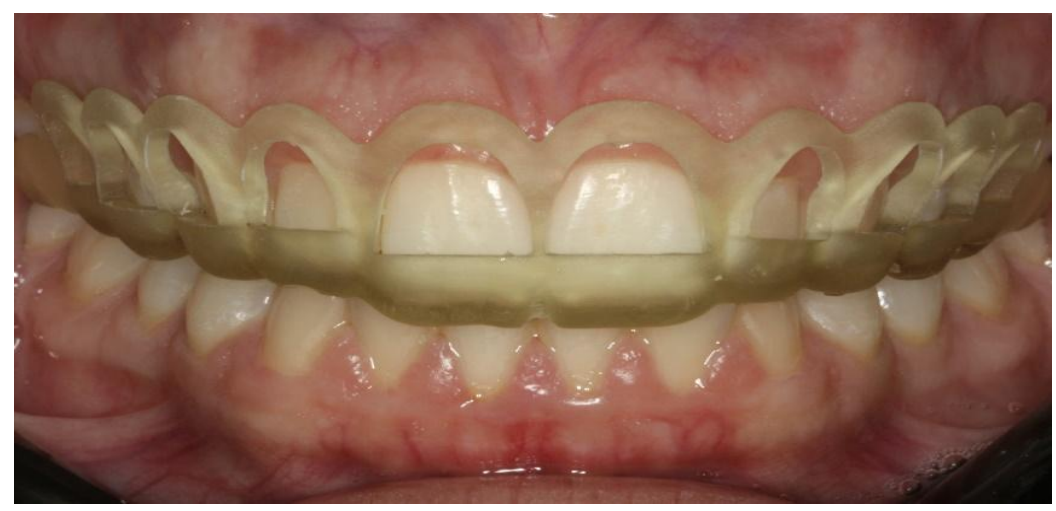

Fonte: Autores. 
Research, Society and Development, v. 10, n. 2, e32111225856, 2022

(CC BY 4.0) | ISSN 2525-3409 | DOI: http://dx.doi.org/10.33448/rsd-v10i2.25856

Figura 5 - Incisão.

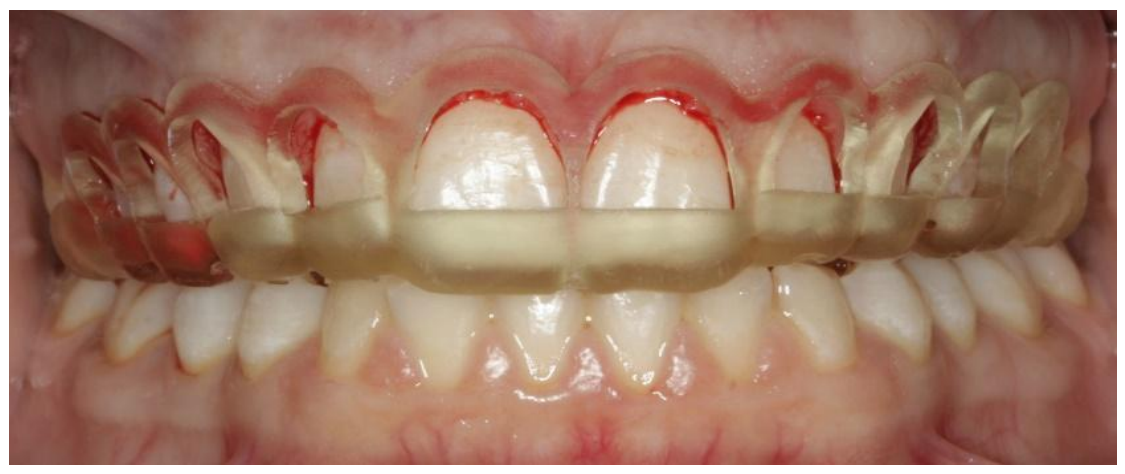

Fonte: Autores.

Figura 6 - Aspecto após remoção do excesso gengival.

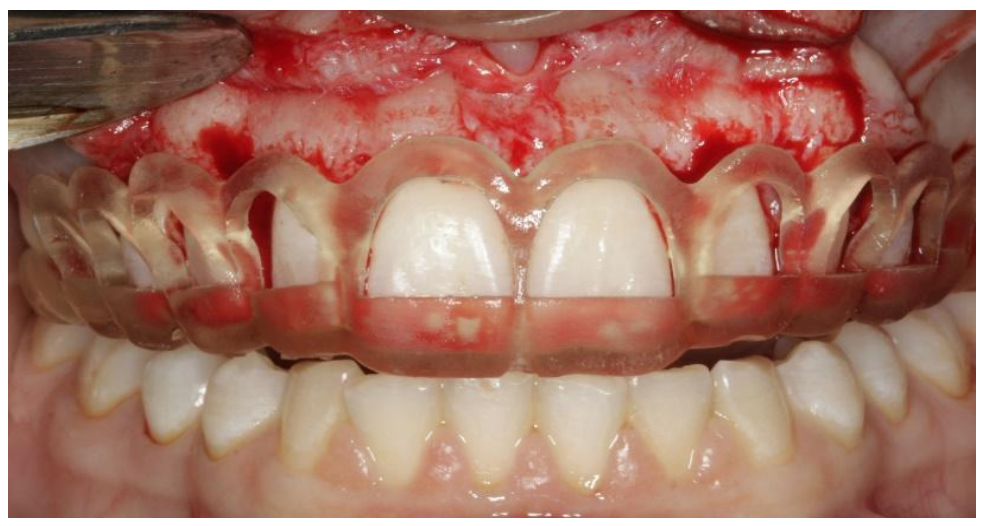

Fonte: Autores.

Figura 7 - Osteotomia.

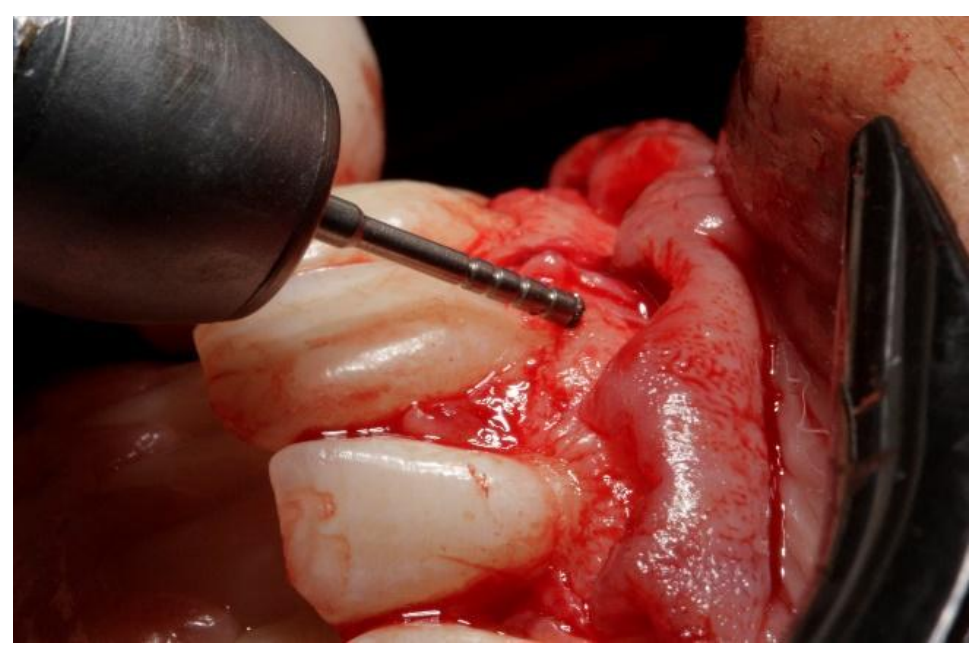

Fonte: Autores. 
Figura 8 - Sutura.

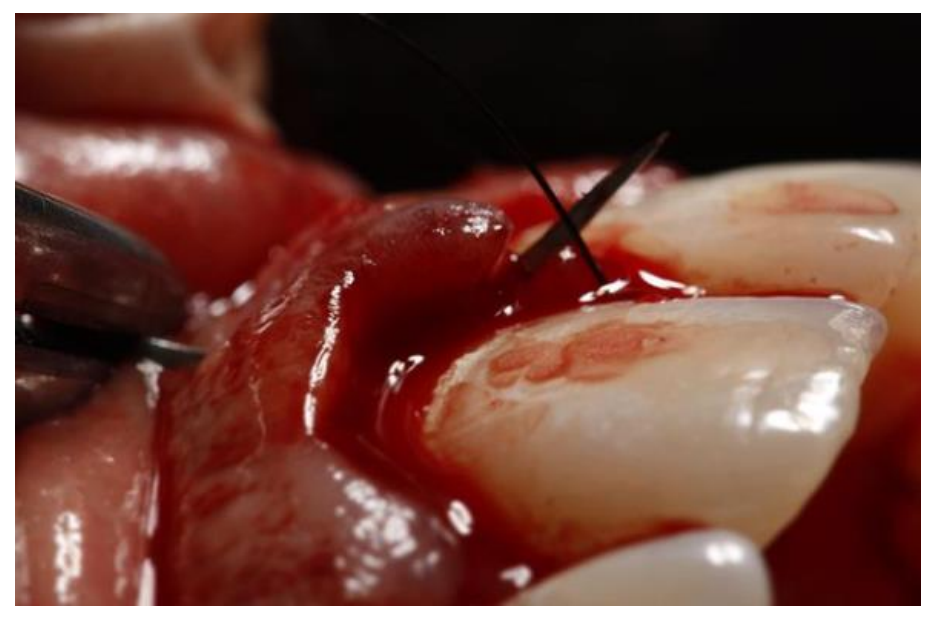

Fonte: Autores.

Figura 9 - Pós operatório Imediato.

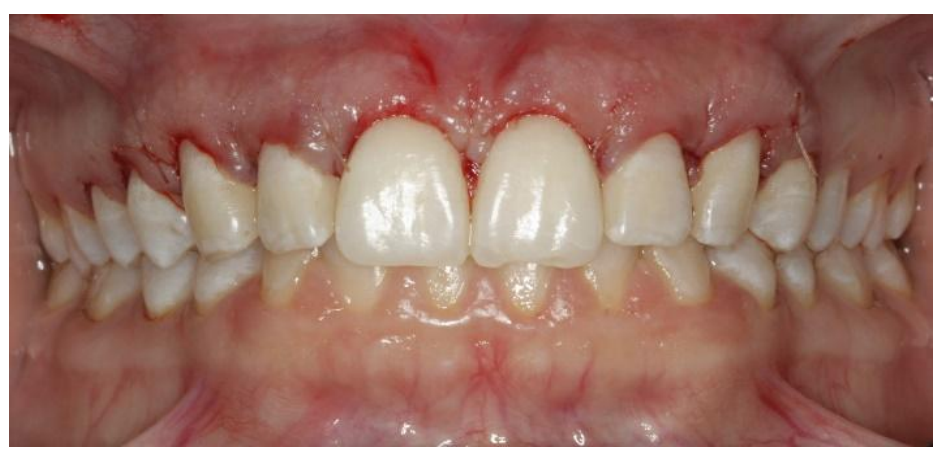

Fonte: Autores.

Na fase pós-operatória foi necessário uso de Dipirona $500 \mathrm{mg}, 01$ comprimido a cada 6 horas, por 3 dias, e para higienização Clorexidina 0,12\% sem álcool, 2 vezes ao dia por 7 dias e na região dos pontos Perioxidin gel. A paciente retornou à clínica para controle pós-operatório 7 dias após a cirurgia, foi constatado perfeita saúde periodontal.

\section{Resultados e Discussão}

Os artigos publicados por Souza et al. (2018), Longo et al. (2019), Nunes. (2020), Ferreira (2020), Deliberador et al. (2020) e Deliberador et al. (2020) sobre a utilização do guia cirúrgico periodontal, concordam que o uso do perioguide diminui o trauma, reduz o tempo de tratamento, acelera a reabilitação acelerando a cicatrização inicial, aumenta o conforto do paciente e oferece estabilidade e previsibilidade a longo prazo com resultados estéticos favoráveis. Diante disto foi decidido orientar a discussão a partir dos seguintes aspectos: previsibilidade, redução de tempo operatório e segurança, visando relacionar e facilitar uma compreensão ampla dos estudos revisados.

\subsection{Previsibilidade}

Nunes et al. (2020), relataram em seus estudos que a cirurgia para correção da estética do sorriso possui uma complexidade elevada, sendo assim tendo ligação com fatores como a posição e tamanho, tanto da estética branca como da rosa, tais quais tornam-se uma dificuldade a mais para o cirurgião-dentista. Diante disso, vê-se a necessidade de um planejamento integrado para que se tenha uma melhor previsibilidade e segurança durante a cirurgia. Eles ainda reforçam que 
com a confecção do guia cirúrgico periodontal é possível adquirir uma melhor comunicação entre o paciente e o cirurgiãodentista, conseguindo assim uma previsão de resultado esperado.

Deliberator et al. (2020) afirmam que novas tecnologias na área odontológica estão abrindo espaço para a variação de técnicas clássicas, visando alcançar resultados mais previsíveis e técnicas menos traumáticas. Os guias cirúrgicos são oriundos da implantodontia e possuem bastante relevância na odontologia por proporcionarem segurança na execução, agilidade e conforto para o paciente durante todo o processo de tratamento. Conclui-se que o uso do guia cirúrgico periodontal multifuncional torna o tratamento mais previsível e compatível com as expectativas do paciente.

\subsection{Redução de tempo operatório}

Ferreira (2020), apresenta que um dos problemas estéticos na desarmonia do sorriso é a exposição excessiva gengival bem como alterações nas proporções dentárias provocadas por diminuição da coroa clínica, que podem ser muitas vezes contornadas. Os autores constataram que a gengivectomia pode auxiliar no equilíbrio das proporções dentárias utilizando o guia cirúrgico. Concluiu-se que a guia cirúrgica facilitou o transoperatório, após verificação da adaptação em boca, auxiliou no corte do tecido gengival tornando este mais preciso e exato.

\subsection{Segurança}

Souza et al. (2018) afirmam em seu estudo que o sorriso é o cartão de visita das pessoas, onde encontramos o que a pessoa quer transmitir e que a harmonização do sorriso é encontrada quando há o equilíbrio entre os dentes, os lábios e as gengivas. Os autores tiveram como objetivo exemplificar através de um caso clínico uma correção do sorriso gengival através do procedimento cirúrgico de gengivectomia e gengivoplastia feito com planejamento digital do sorriso. Concluíram que o uso do guia cirúrgico é satisfatório pois diminui o tempo de cirurgia, facilita para o profissional e dá segurança ao paciente.

Longo et al. (2019) exemplifica que uma exibição gengival excessiva, influencia a aparência do sorriso e o comportamento social dos pacientes, sendo erupção passiva alterada (EPA) o fator mais comum. Os autores buscaram relatar a nova abordagem para o tratamento de EPA com a utilização da guia cirúrgica periodontal através de um relato de caso clínico. Após finalização do procedimento e acompanhamento durante 6 meses, os autores concluíram que os guias digitais personalizadas podem ser usados com segurança para procedimentos clínicos de alongamento de coroa em pacientes afetados por EAP, reduzindo defeitos de recessão ou rebote de tecido mole nas fases inicial e tardia da cicatrização e aumentando a precisão do desenho do retalho. Porém ressaltam que mais estudos com acompanhamento mais longo são necessários para confirmar esse achado.

Em outro estudo Deliberator et al. (2020) afirmam que um sorriso perfeito é ditado por um equilíbrio entre três parâmetros: dentes, gengiva e lábios. Com o aumento significativo na procura de cirurgias estéticas e menos traumáticas, o fluxo digital oferece ao clínico uma ferramenta diagnóstica para fazer a escolha do procedimento mais adequado e permite um planejamento de tratamento com maior previsibilidade e segurança. Concluíram que com o uso do perioguide temos um procedimento mais rápido, preciso e seguro, assim, tornando o tratamento mais previsível e compatível com as expectativas do paciente.

\section{Conclusão}

Em virtude dos fatos mencionados, é possível concluir que a atuação interdisciplinar entre as especialidades da Periodontia com a Radiologia auxiliada com a tecnologia possibilita aperfeiçoar e otimizar a cirurgia de gengivoplastia/gengivectomia. $\mathrm{O}$ uso do perioguide foi um diferencial para sucesso do tratamento. 
Verificou-se que as cirurgias realizadas com o uso do guia são mais eficientes, apresentando um melhor desempenho quando comparado à técnica cirúrgica convencional, demonstrando que o mesmo facilita o ato cirúrgico, permitindo maior previsibilidade e com incisões suficientemente invasivas, possibilitando uma recuperação pós operatória menos dolorosa, adianta a reabilitação acelerando o processo de cicatrização e o aumento do conforto do paciente.

Vale ainda ressaltar que as únicas desvantagens encontradas no guia seriam seu custo elevado para confecção e a necessidade de um trabalho multidisciplinar, limitando, na atualidade, o acesso ao dispositivo por todo e qualquer paciente.

\section{Referências}

Deliberador, T. M., Weiss, S. G., Neto, A. T. D., Zetola, I. Z., Prix, M. E .S.,Junior, D. R., Martins, H. H., Storrer, C. L. M. (2020). Mucoguide Para Túnel Subepitelial Enxerto De Tecido Conjuntivo: Um Novo Abordagem Cirúrgica. Tecnologias Aplicadas à Prática e ao Ensi no da Odontologia.

Deliberador, T. M., Weiss, S. G., Neto, A. T. D., Zetola, I. Z., Prix, M. E. S.,Junior, D. R., Martins, H. H., Storrer, C .L. M. (2020). Guided Periodontal Surgery: Association of Digital Workflow and Piezosurgery for the Correction of a Gummy Smile. Case Rep Dent, 1-6.

Ferreira, J. F. S. C. (2020) A Gengivectomia Como Ferramenta No Equilíbrio Das Proporções Dentárias: Revisão Narrativa A Propósito De Um Caso Clínico. Dissertação Mestre em Medicina Dentária, Universidade Católica Portugues, Viseu(Portugal).

Januário, A. L., Barriviera, M., Duarte, W. R. (2008). Soft tissue cone-beam computed tomography: a novel method for the measurement of gingival tissue and the dimensions of the dentogingival unit. Journal of esthetic and restorative dentistr,.20(6).

Joly, J. C., da Silva R.C, de Carvalho, P.C.M. Reconstrução Tecidual Estética capítulo 1; Editora artes médicas SP. 2010.

KahN, S., Dias, A. Sorriso Gengival. $1^{\text {a }}$ Edição. Quintessence Nacional, 2017. Cap. 1, p. 4.

Kan, J. Y., Morimoto, T., Rungcharassaeng, K., Roe, P., Smith, D. H. (2010). Gingival biotype assessment in the esthetic zone: visual versus direct measurement. The International journal of periodontics \& restorative dentistry, 30(3), 237-243.

Longo, E., Frosecchi, M., Marradi, L., Signore, A., \& de Angelis, N. (2019). Guided periodontal surgery: a novel approach for the treatment of gummy smile. A case report. The international journal of esthetic dentistry, 14(4), 384-392.

Nunes, I. S., Lacerda, J. C. F., de Nogueira, P. L., Dantas, M. V. O., Ribeiro, R. A., Rodrigues, R. de Q. F., Sousa, J. N. L. (2020). Development of surgical guidelines for auxiliary technique of gingivectomy on inner bevel with osteotomy: report case. Research, Society and Development, 9(7), e70973923.

Souza, N .C., Cornélio, A. L. G., Gaze, V. A. M. (2018). Gengivoplastia com guia cirúrgico - Correção sorriso gengival - Relato de caso clínico. R Odontol Planal Cent.

Souza, M. A., Vieira, R. M., Gonçalves, V. P. D., Azevedo, M. M. A., Feghali, F. J. (2020). Administração de drogas em Odontologia do esporte e o risco de doping. Full Dent. Sci, 11(43):64-68

Zweers, J., Thomas, R. Z., Slot, D. E., Weisgold, A. S., Van der Weijden, F. G. (2014). Characteristics of periodontal biotype, its dimensions, associations and prevalence: a systematic review. Journal of clinical periodontology, 41(10), 958-971. 